\title{
Predictive Risk Factors Associated With Increased Opioid Use Among Patients Undergoing Elective Spine Surgery
}

\author{
ZACHARY SANFORD, MD,${ }^{1}$ ANDREW BRODA, BS, ${ }^{1}$ HALEY TAYLOR, MS $2,{ }^{1}$ JUSTIN TURCOTTE, PHD, \\ MBA, ${ }^{2}$ CHAD M. PATTON, MD, MS ${ }^{1,3}$ \\ ${ }^{1}$ Center for Spine Surgery, Anne Arundel Medical Center, Annapolis, Maryland, ${ }^{2}$ Department of Surgery, Anne Arundel Medical Center, Annapolis, Maryland, \\ ${ }^{3}$ Orthopedic and Sports Medicine Specialists, Anne Arundel Medical Center, Annapolis, Maryland
}

\begin{abstract}
Background: Opioids are commonly used for postoperative pain management in spine surgery. However, few guidelines exist for appropriate prescribing in the acute postoperative phase of care. We identify risk factors for inpatient (IP) opioid use and examine relationships between IP requirements and discharge (DC) opioid prescriptions.

Methods: Retrospective review of elective spine surgeries between January 2014 and May 2018 identified cases of lumbar decompression (LD), LD with fusion (LDF), and cervical decompression with fusion (CDF) at our high-volume spine center. Multiple regression examining potential risk factors for opioid use was performed. Opioid use was normalized into daily morphine milligram equivalents (MME).

Results: A total of 2281 patients who underwent $1251 \mathrm{LD}, 384 \mathrm{LDF}$, and $648 \mathrm{CDF}$ procedures were identified $\left(54.1 \%\right.$ male, mean age $=57.9$ years, mean body mass index $=30.3 \mathrm{~kg} / \mathrm{m}^{2}$, median American Society of Anesthesiologists [ASA] score = 2). Mean IP opioid use was $44.4 \mathrm{MME}$ /day and average DC prescriptions totaled 496.5 MME. Multiple regression models identified younger age and increased ASA score as predictive of increased daily IP opioid consumption $\left(\beta_{\mathrm{AGE}}=-0.36, P<.001, \beta_{\mathrm{ASA}}=10.1, P<.001 ; R^{2}=0.308\right)$ and increased DC opioid amounts $\left(\beta_{\mathrm{AGE}}=-4.62, P<.001, \beta_{\mathrm{ASA}}=72.1, P<.001 ; R^{2}=0.097\right)$. Highest IP and DC opioid use was observed among LDF followed by CDF and LD patients. Significant positive correlations were found between IP opioid usage and DC opioid prescriptions by IP opioid quartiles ( $r=0.99 \mathrm{LD}, 0.98 \mathrm{LDF}, 0.96 \mathrm{CDF})$.

Conclusions: Younger patients and higher ASA scores correlated with increased IP opioid use and DC opioid prescriptions. DC prescriptions appropriately reflect IP use.

Level of Evidence: 3.

Clinical Relevance: Adequate pain management is an integral component to successful outcomes in spine surgery. Awareness of candidates likely to require higher levels of opioid analgesia will be beneficial in guiding surgeon prescribing practices.

Complications
\end{abstract}

Keywords: opioid, spine surgery, predictive modeling, risk factors, lumbar decompression, cervical decompression, fusion

\section{INTRODUCTION}

The use of opioid analgesia has increased following the introduction of the Pain as the Fifth Vital Sign campaign of the mid-1990s. ${ }^{1,2}$ Concurrently, international collaborations such as those published in the Lancet as part of the 2013 Global Burden of Disease Study have identified chronic back and neck pain as a leading cause of disabilityadjusted life-years lost worldwide. ${ }^{3}$ Patients suffering from such complex pain syndromes often experience impairment in function and quality of life, usually requiring multimodal treatment options which may include opioid medication management. Formerly reserved for intractable pain control among the terminally ill, opioid medications have since become a common tool in managing painful spine-related conditions including postoperative pain.

In parallel, the volume of spine surgeries has grown substantially in recent years and surgical treatment has been shown to have favorable outcomes across a wide range of spinal pathologies. ${ }^{4,5}$ However, as the frequency of spine surgeries continue to increase, it is important to understand the role of pharmacological pain management in the restoration of normal function to these patients. ${ }^{6}$ This is especially true in light of the current opioid epidemic as policy makers act to throttle prescriptions while patient perceptions of pain control have 
become incorporated into satisfaction scores used as a surrogate measure of hospital quality. ${ }^{7}$

Strategies for postoperative pain management have evolved over time. Previous attempts relied on patient-controlled intravenous analgesia (PCA), but anecdotal evidence and published reports suggest that the use of PCA increases hospital length of stay. ${ }^{8}$ More recent approaches include a variety of multimodal oral analgesic agents. ${ }^{9,10}$ When ordering postoperative medications, surgeons and care teams consider several factors including patient age, medical comorbidities, surgical invasiveness, and prior medication use. However, there is no agreed upon standard for managing pain following specific spine-related procedures. Furthermore, little is published regarding risk factors for inpatient (IP) opioid use or prescription pain control needs at discharge (DC) for these patients. The goal of our study was to identify risk factors for IP opioid use following 3 common spine surgeries and examine the relationship between IP opioid use and DC prescriptions.

\section{MATERIALS AND METHODS}

\section{Data Collection}

This study was deemed institutional review board exempt by the institutional clinical research committee. This investigation used internal data analyzed retrospectively from spine surgery cases performed at a single institution with a dedicated spine surgery IP unit.

\section{Data of Interest}

Patient demographics included age, sex, and body mass index (BMI). Indices of patient comorbidities included the American Society of Anesthesiologists (ASA) score and the Centers for Medicare and Medicaid Services Hierarchical Conditions Category (CMS-HCC) score, previously used to stratify financial risk in Medicare Advantage patients. ${ }^{11}$ Opioid data included postoperative IP opioid use and amount of opioid prescribed at DC based on available electronic medical record data and normalized into morphine milligram equivalents (MMEs).

\section{Inclusion and Exclusion Criteria}

Our database included information on over 5000 spinal surgery cases based on an internal query of patients undergoing spine surgery between January
Table 1. Conversion chart of morphine milligram equivalents (MMEs). Conversions are those presented by the Centers for Disease Control and Prevention. ${ }^{12}$

\begin{tabular}{lc}
\hline Opioid & Conversion Factor \\
\hline Codeine/acetaminophen & 0.2 \\
Tapentadol & 0.4 \\
Hydrocodone/acetaminophen & 1 \\
Morphine & 1 \\
Oxycodone & 1.5 \\
Oxycodone/acetaminophen & 1.5 \\
Fentanyl transdermal & 2.4 \\
Oxymorphone & 3 \\
Hydromorphone & 4 \\
Methadone, mg/d & \\
1-20 & 4 \\
$21-40$ & 8 \\
$41-60$ & 10 \\
$61-80$ & 12 \\
\hline
\end{tabular}

2014 and May 2018. Patients were categorized by procedure type and included lumbar decompression (LD), LD and fusion (LDF), or cervical discectomy with fusion (CDF). The database includes both primary and revision surgical procedures.

\section{Opioid Use}

IP opioid consumption was calculated from all narcotic orders placed during the duration of individual patients' length of stay (LOS) and subsequently normalized into daily usage as a function of recorded LOS. Opioid medications prescribed at time of DC were quantified as those prescriptions written at DC for patients undergoing the identified spinal surgery procedure. IP opioid use and opioids prescribed at DC were normalized into MMEs in accordance with the Centers for Disease Control and Prevention's established guidelines (Table 1). ${ }^{12}$ All opioid use data were stratified into quartiles by MME for subsequent analysis.

\section{CMS-HCC Score Calculation}

The CMS-HCC score was used in this study as a possible predictor of opioid use. The publicly available scoring methodology, originally designed to predict financial risk in Medicare Advantage patients, was replicated and used to generate an objective risk score for our series of patients. In brief, data are scored using health plan enrollee diagnoses and demographic data in order to predict medical expenditure risk, from which a risk adjustment model is able to identify high- and low-cost individuals based on established hierarchical categories. ${ }^{11}$ Our previous work and forthcom- 
Sanford et al.

Table 2. Summary of descriptive statistics for patients undergoing elective spine surgery at our center. Data reported as mean \pm standard error.

\begin{tabular}{|c|c|c|c|c|c|}
\hline & All Procedures & $\begin{array}{c}\text { Lumbar } \\
\text { Decompression }\end{array}$ & $\begin{array}{c}\text { Lumbar Decompression } \\
\text { with Fusion }\end{array}$ & $\begin{array}{c}\text { Cervical Decompression } \\
\text { with Fusion }\end{array}$ & $\boldsymbol{P}$ \\
\hline $\mathrm{N}$ & 2281 & 1251 & 384 & 648 & \\
\hline Age, y & $57.9 \pm 0.3$ & $57.9 \pm 0.5$ & $61.2 \pm 0.6$ & $56.0 \pm 0.5$ & $<.001^{\mathrm{a}}$ \\
\hline Sex, male/female, $\%$ & $54 / 46$ & $59 / 41$ & $49 / 51$ & $48 / 52$ & $<.001^{\mathrm{a}}$ \\
\hline BMI, $\mathrm{kg} / \mathrm{m}^{2}$ & $30.3 \pm 0.13$ & $30.1 \pm 0.17$ & $30.6 \pm 0.3$ & $30.4 \pm 0.2$ & .73 \\
\hline ASA score & $2.4 \pm 0.01$ & $2.3 \pm 0.02$ & $2.5 \pm 0.03$ & $2.4 \pm 0.02$ & $<.001^{\mathrm{a}}$ \\
\hline CMS-HCC score & $0.82 \pm 0.02$ & $0.77 \pm 0.02$ & $0.93 \pm 0.05$ & $0.80 \pm 0.03$ & $<.001^{\mathrm{a}}$ \\
\hline Daily inpatient opioid, MME & $44.3 \pm 1.3$ & $19.7 \pm 0.9$ & $105.3 \pm 4.1$ & $56.0 \pm 2.0$ & $<.001^{\mathrm{a}}$ \\
\hline Prescribed discharge opioid, MME & $496 \pm 8$ & $406 \pm 8$ & $595 \pm 23$ & $611 \pm 18$ & $<.001^{\mathrm{a}}$ \\
\hline
\end{tabular}

Abbreviations: ASA score, American Society of Anesthesiologists score; BMI, body mass index; CMS-HCC Score, Centers for Medicare and Medicaid Services Hierarchical Conditions Category score; MME, morphine milligram equivalents.

${ }^{\mathrm{a}}$ Statistical significance $(P<.05)$.

ing studies suggest that this score may have clinical use for care teams.

\section{STATISTICAL METHODS}

\section{Statistical Analysis}

All statistical analyses were performed using MathWorks MATLAB version R2017b. Patient cohorts were compared using 1-way analysis of variance (ANOVA) and Pearson $\chi^{2}$ analysis to establish significant differences between groups. Inherent difference between the 3 procedure groups of study are controlled through including a procedure variable in further statistical analyses.

Multiple linear regression analysis was performed to examine predictors of both IP daily opioid consumption and amount of opioid medication prescribed at DC. Predictor variables including age, BMI, ASA score, and CMS-HCC score were assessed for relationship with both outcome variables. IP daily opioid consumption was included in the multiple linear regression model as a potential predictor of DC opioid amounts.

\section{Quartile Analysis}

Patients were stratified into quartiles based on IP daily opioid consumption and opioid medication amounts prescribed at DC. One-way ANOVA was performed on IP opioid consumption quartiles against DC opioid prescriptions controlling for procedure type. Correlation analysis was also performed between opioids dispensed during hospitalization and those prescribed at time of DC.

\section{RESULTS}

\section{Patient Demographics}

We identified 2281 patients during a 53-month interval between January 2014 and May 2018 who underwent elective LD, LDF, or CDF at our center (Table 2). Patients exhibited similar BMI values across all procedures with an average of $30.3 \mathrm{~kg} / \mathrm{m}^{2}$. Significant differences were found across cohorts by age, sex, ASA score, and CMS-HCC score $(P<$ $.001)$. Patients undergoing LDF tended to be older $(\mathrm{LDF}=61.2$ years, $\mathrm{LD}=57.9$ years, $\mathrm{CDF}=56.0$ years) and were determined to have higher ASA and CMS-HCC scores $(\mathrm{LDF}=2.5 / 0.93, \mathrm{LD}=2.3 / 0.77$, $\mathrm{CDF}=2.4 / 0.80)$ compared with the other surgical cohorts. A larger proportion of patients undergoing $\mathrm{LD}$ at our center were male $(\mathrm{LD}=54 \%, \mathrm{LDF}=$ $49 \%, \mathrm{CDF}=48 \%$ ).

\section{Opioid Use}

LD patients consumed lower average daily amounts of opioid medications during IP hospitalization $(\mathrm{LD}=19.7 \mathrm{MME}, \mathrm{LDF}=105.3 \mathrm{MME}$, $\mathrm{CDF}=56.0 \mathrm{MME}, P<.001)$ and average total amounts at time of DC (LD = $406 \mathrm{MME}, \mathrm{LDF}=$ $595 \mathrm{MME}, \mathrm{CDF}=611 \mathrm{MME}, P<.001)$ as compared with the LDF or CDF cohorts (Table 2). Opioid use varied across procedures for both IP daily opioid consumption and DC prescriptions (Figure 1). Among the IP cohorts, average daily opioid consumption ranged from 0 to 754.6 MME. By procedure, $\mathrm{LD}_{\mathrm{IP}}$ demonstrated median (interquartile range) of $10 \mathrm{MME}$ (5-23 MME) compared with $\mathrm{LDF}_{\mathrm{IP}}=85.7 \mathrm{MME}(60-127.4 \mathrm{MME})$ and $\mathrm{CDF}_{\mathrm{IP}}=44.2 \mathrm{MME}$ (23.9-73.7 MME; Table 3). At DC, prescription totals included amounts from 0 to 5400 MME, with $\mathrm{LD}_{\mathrm{DC}}=300$ MME (225-450 $\mathrm{MME}), \mathrm{LDF}_{\mathrm{DC}}=450 \mathrm{MME}(375-675 \mathrm{MME})$, and $\mathrm{CDF}_{\mathrm{DC}}=480 \mathrm{MME}(337.5-750 \mathrm{MME})$. From these data, observable trends illustrated strong linearity, with coefficients of correlation for relatedness between daily IP opioid amounts and prescriptions at time of DC greater than $90 \%$ across all quartiles and procedures. (LD $R=0.93, \mathrm{LDF} R=0.96$, CDF 


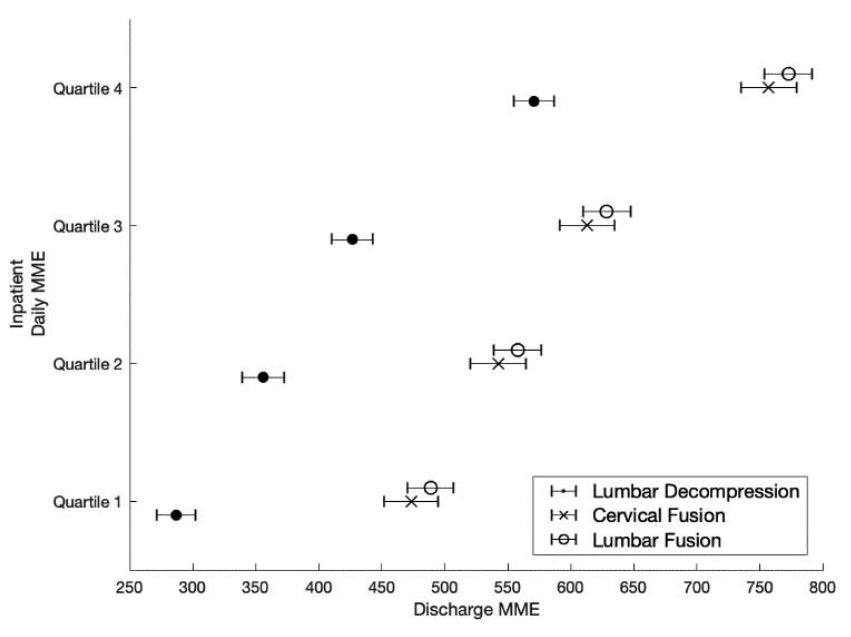

Figure 1. Discharge opioid prescriptions for lumbar decompression, lumbar decompression with fusion, and cervical decompression with fusion stratified by inpatient daily opioid consumption (quartiles). MME, morphine milligram equivalent.

$R=0.96$ ). Statistically significant relatedness was demonstrated between daily IP opioid consumption and outpatient DC prescriptions for LDF and CDF (LDF $P=.043$, CDF $P=.003)$ but not for $\operatorname{LD}(P=$ $.068)$.

\section{Statistical Modeling}

Multiple linear regression analysis was performed to assess demographic and preoperative variables to postoperative opioid use in both the IP and outpatient setting while controlling for procedure type (Table 4). Age and ASA score were found to be statistically predictive of opioid use during hospitalization. Increased IP daily opioid use was predicted in younger patients, with a decrease of $0.36 \mathrm{MME}$ per additional year of age $(\beta=-0.36$, $95 \%$ confidence interval $[\mathrm{CI}]=-0.52$ to $-0.19, P<$ $.001)$ and an increase of $10.1 \mathrm{MME}$ per point increase in ASA score $(\beta=10.1,95 \% \mathrm{CI}=5.95$ to 14.17, $\left.P<.001, R^{2}=0.308\right)$. With regard to amounts of opioid medications prescribed at time of DC, modeling demonstrated age, ASA score, and IP daily opioid consumption to be significant predictors of DC opioid amounts. Increases in amount of prescribed DC opioid medication were associated with younger patients at a rate of $3.88 \mathrm{MME}$ per year of age $(\beta=-3.88,95 \% \mathrm{CI}=-5.04$ to $-2.72, P$ $<.001$ ), and increase of $51.11 \mathrm{MME}$ per 1 point increase in ASA score $(\beta=51.11,95 \% \mathrm{CI}=21.62$ to 80.60, $P \leq .001)$. Relatedness between daily amounts of IP opioid medications while hospitalized and DC prescriptions demonstrated a 2:1 relationship, with patients receiving approximately twice the amount of opioid medication at time of DC for each additional MME received during an average day of hospitalization $(\beta=2.08,95 \% \mathrm{CI}=1.79$ to $2.38, P$ $\left.<.001, R^{2}=0.168\right)$.

\section{DISCUSSION}

Previous literature has investigated the relationship between baseline opioid use and sustained longterm opioid usage. Work from the Spine Patient Outcomes Research Trial (SPORT) cohort of 2,110 patients demonstrated significant risk factors associated with baseline opioid use including young age, lack of college education, unemployment, disability status, smoking history, and concurrent mental disorders. ${ }^{13}$ The relationship between prior opioid usage and long-term continuation following spinal surgery has also been reported. ${ }^{14-17}$ This is in agreement with the well-studied potentiation of analgesia through $\mu$-opioid receptor agonism which has been clearly and repeatedly demonstrated to possess substantial addictive potential. ${ }^{18,19}$

Preoperative chronic opioid use itself presents substantial risks to postoperative spine surgery outcomes. Specifically, heavy opioid users are at significant risk for increased 90-day wound complications and pain disorders, culminating in increased returns to the emergency department and readmission to the hospital as well as continued postoperative use. ${ }^{20}$ Similar trends have been observed in

Table 3. Quartile analysis of opioids dispensed at inpatient and discharge phases of care. Data are reported in average opioid amount (MME) per quartile with accompanying low and high values.

\begin{tabular}{|c|c|c|c|c|c|c|c|}
\hline \multirow[b]{2}{*}{ Procedure } & \multirow{2}{*}{$\begin{array}{l}\text { Phase } \\
\text { of Care }\end{array}$} & \multicolumn{4}{|c|}{ Interquartile Range } & \multirow[b]{2}{*}{$R$} & \multirow[b]{2}{*}{$\boldsymbol{P}$} \\
\hline & & Quartile 1 & Quartile 2 & Quartile 3 & Quartile 4 & & \\
\hline \multirow{2}{*}{$\begin{array}{l}\text { Lumbar decompression, mean } \\
\text { (quartile range) }\end{array}$} & Inpatient & $1.3(0-5)$ & $7.5(5-10)$ & $15.3(10-23)$ & $56.2(23-491.0)$ & \multirow[t]{2}{*}{0.93} & \multirow[t]{2}{*}{.068} \\
\hline & Discharge & $168.9(0-225)$ & $296.5(225-300)$ & $432.7(300-450)$ & $801.9(450-2025)$ & & \\
\hline \multirow{2}{*}{$\begin{array}{l}\text { Lumbar decompression with fusion, } \\
\text { mean (quartile range) }\end{array}$} & Inpatient & $38.6(0-60)$ & $73.8(60-85.7)$ & $104.0(85.7-127.4)$ & $206.8(127.4-754.6)$ & \multirow[t]{2}{*}{0.96} & \multirow[t]{2}{*}{$.043^{\circ}$} \\
\hline & Discharge & $244.0(0-375)$ & $445.4(375-450)$ & $599.7(450-675)$ & $1253.5(675-3,375)$ & & \\
\hline \multirow{2}{*}{$\begin{array}{l}\text { Cervical decompression with fusion, } \\
\text { mean (quartile range) }\end{array}$} & Inpatient & $12.0(0-23.9)$ & $33.9(23.9-44.2)$ & $56.8(44.2-73.7)$ & $121.4(73.7-527.0)$ & \multirow[t]{2}{*}{0.96} & \multirow[t]{2}{*}{$.003^{\circ}$} \\
\hline & Discharge & $240.7(0-337.5)$ & $447.4(337.5-480)$ & $649.5(480-750)$ & $1180.8(750-5,400)$ & & \\
\hline
\end{tabular}

${ }^{\text {a }}$ Statistical significance $(P<.05)$. 
Table 4. Multiple regressions for patient demographic data controlling for spine surgery by procedure. Inpatient opioid consumption reported in $M M E / d$ $\left(R^{2}=0.308\right)$. Prescribed opioid amounts at discharge reported in $\mathrm{MME}\left(R^{2}=\right.$ 0.168).

\begin{tabular}{lccc}
\hline Parameter & $\boldsymbol{\beta}$ & $\mathbf{9 5 \%} \mathbf{C I}$ & $\boldsymbol{P}$ \\
\hline Daily inpatient opioid & consumption, & MME & \\
Age & -0.36 & -0.52 to -0.19 & $<.001^{\mathrm{a}}$ \\
ASA & 10.1 & 5.95 to 14.17 & $<.001^{\mathrm{a}}$ \\
BMI & -0.07 & -0.43 to 0.29 & .72 \\
CMS-HCC & 0.54 & -1.94 to 3.02 & .67 \\
Opioids prescribed at discharge, & MME & \\
Age & -3.88 & -5.04 to -2.72 & $<.001^{\mathrm{a}}$ \\
ASA & 51.11 & 21.62 to 80.60 & $<.001^{\mathrm{a}}$ \\
BMI & -0.55 & -3.13 to 2.02 & .67 \\
CMS-HCC & -9.94 & -27.64 to 7.77 & .27 \\
MME/d & 2.08 & 1.79 to 2.38 & $<.001^{\mathrm{a}}$ \\
\hline
\end{tabular}

Abbreviations: ASA score, American Society of Anesthesiologists score; BMI, body mass index; CI, confidence interval; CMS-HCC Score, Centers for Medicare and Medicaid Services Hierarchical Conditions Category score; MME, morphine milligram equivalent.

${ }^{\text {a }}$ Statistical significance $(P<.05)$.

instances of worker's compensation cases, where opioid use prior to cervical fusion was correlated to inferior return to work, reoperation rate, and permanent disability outcomes. ${ }^{21}$ Survey instruments designed to identify chronic baseline opioid use prior to surgery have been effective at predicting suboptimal patient reported outcomes and may play a role in helping to augment how surgeons plan postoperative follow up or counseling. ${ }^{22}$ Identifying these risks prior to surgical treatment may allow treatment teams to allocate resources designed to optimize patient outcomes in the acute perioperative period.

Our findings suggest that ASA score and age are associated with anticipated increases in opioid medication both during the hospital encounter and at time of DC. Increasing ASA score was linked with higher IP and DC opioids, while increasing age is inversely related to their opioid use. This observation may be explained by age-related differences in pain perception or caution in prescribing higher doses of opioids in older patients. However, independent of underlying comorbidities, there remains risk that younger patients will consume greater amounts of opioid medications throughout their postoperative course. These risk factors may potentially serve as useful adjunctive markers to known related comorbidities linked to long-term opioid usage following spine surgery such as migraine disorders, diabetes, chronic depression, and smoking. ${ }^{23}$

Our data also serve as a first step in developing standardized, appropriate opioid prescriptions at time of DC from specific and common spine procedures. DC prescriptions appear to reflect actual IP opioid use rather than arbitrary and often large amounts of opioids. Based on our findings, we suggest that IP opioid use may be an accurate means of assessing potential outpatient needs during the immediate postoperative period.

Our study was limited to data in our electronic medical records. While this accurately depicts IP use and DC prescriptions, capturing additional refills or actual outpatient consumption was beyond the scope of this study, although additional work is currently underway in order to track individual patient use and refills beyond DC.

\section{CONCLUSIONS}

Our analysis demonstrates younger patients and higher ASA scores are associated with greater IP and DC opioid use. In addition, DC prescriptions reflect IP use. Further study is underway to track postoperative prescription refills and use beyond the initial DC prescription. Ultimately, this will help surgeons develop refined, procedure-specific prescribing protocols.

\section{REFERENCES}

1. Scher C, Meador L, Van Cleave JH, Reid MC. Moving beyond pain as the fifth vital sign and patient satisfaction scores to improve pain care in the 21st century. Pain Manag Nurs. 2018;19(2):125-129.

2. Tompkins DA, Hobelmann JG, Compton P. Providing chronic pain management in the "fifth vital sign" era: historical and treatment perspectives on a modern-day medical dilemma. Drug Alcohol Depend. 2017;173(Suppl 1):S11-S21.

3. DALYs GBD, Collaborators H, Murray CJ, et al. Global, regional, and national disability-adjusted life years (DALYs) for 306 diseases and injuries and healthy life expectancy (HALE) for 188 countries, 1990-2013: quantifying the epidemiological transition. Lancet. 2015;386(10009):2145-2191.

4. Weinstein JN, Lurie JD, Tosteson TD, et al. Surgical compared with nonoperative treatment for lumbar degenerative spondylolisthesis. four-year results in the Spine Patient Outcomes Research Trial (SPORT) randomized and observational cohorts. J Bone Joint Surg Am. 2009;91(6):1295-1304.

5. Weinstein JN, Tosteson TD, Lurie JD, et al. Surgical vs nonoperative treatment for lumbar disk herniation: the Spine Patient Outcomes Research Trial (SPORT): a randomized trial. JAMA. 2006;296(20):2441-2450.

6. Martin B, Mirza SK, Spina N, Spiker WR, Lawrence B, Brodke DS. Trends in lumbar fusion procedure rates and associated hospital costs for degenerative spinal diseases in the United States, 2004-2015. Spine (Phila Pa 1976). 2018;44(5): 369-376.

7. NQF endorses HCAHPS patient perception survey. Healthcare Benchmarks Qual Improv. 2005;12(7):82-83.

8. Van Boerum DH, Smith JT, Curtin MJ. A comparison of 
the effects of patient-controlled analgesia with intravenous opioids versus epidural analgesia on recovery after surgery for idiopathic scoliosis. Spine (Phila Pa 1976). 2000;25(18):23552357.

9. Meng $\mathrm{Y}$, Jiang $\mathrm{H}$, Zhang $\mathrm{C}$, et al. A comparison of the postoperative analgesic efficacy between epidural and intravenous analgesia in major spine surgery: a meta-analysis. $J$ Pain Res. 2017;10:405-415.

10. Hwang CJ, Chung SS, Lee KY, et al. Analgesic efficacy and safety of prolonged-release oxycodone/naloxone in Korean patients with chronic pain from spinal disorders. Clin Orthop Surg. 2018;10(1):33-40.

11. Centers for Medicare and Medicaid Services, Center for Consumer Information and Insurance Oversight. Discussion Paper. HHS-Operated Risk Adjustment Methodology Meeting. March 31, 2016.

12. Dowell D, Haegerich TM, Chou R. CDC guidelines for prescribing opioids for chronic pain-United States, 2016. JAMA. 2016;315(15):1624-1645.

13. Krebs EE, Lurie JD, Fanciullo G, et al. Predictors of long-term opioid use among patients with painful lumbar spine conditions. J Pain. 2010;11(1):44-52.

14. Armaghani SJ, Lee DS, Bible JE, et al. Preoperative opioid use and its association with perioperative opioid demand and postoperative opioid independence in patients undergoing spine surgery. Spine (Phila Pa 1976). 2014; 39(25):E1524-1530.

15. Connolly J 3rd, Javed Z, Raji MA, Chan W, Kuo YF, Baillargeon J. Predictors of long-term opioid use following lumbar fusion surgery. Spine (Phila Pa 1976). 2017; 42(18):1405-1411.

16. Lall MP, Restrepo E. Predictors of weeks to opioid cessation after lumbar fusion: a prospective cohort study. Pain Manag Nurs. 2018;19(5):525-534.

17. O’Donnell JA, Anderson JT, Haas AR, et al. Preoperative opioid use is a predictor of poor return to work in workers' compensation patients after lumbar diskectomy. Spine (Phila Pa 1976). 2018;43(8):594-602.

18. Contet C, Kieffer BL, Befort K. Mu opioid receptor: a gateway to drug addiction. Curr Opin Neurobiol. 2004; 14(3):370-378.

19. Margolis EB, Fields HL. Mu opioid receptor actions in the lateral habenula. PLoS One. 2016;11(7):e0159097.

20. Jain N, Phillips FM, Weaver T, Khan SN. Preoperative chronic opioid therapy: a risk factor for complications, readmission, continued opioid use and increased costs after one- and two-level posterior lumbar fusion. Spine (Phila Pa 1976). 2018;43(19):1331-1338.

21. Faour M, Anderson JT, Haas AR, et al. Preoperative opioid use: a risk factor for poor return to work status after single-level cervical fusion for radiculopathy in a workers' compensation setting. Clin Spine Surg. 2018;31(1):E19-E24.

22. Lee D, Armaghani S, Archer KR, et al. Preoperative opioid use as a predictor of adverse postoperative self-reported outcomes in patients undergoing spine surgery. $J$ Bone Joint Surg Am. 2014;96(11):e89.

23. Qureshi R, Werner B, Puvanesarajah V, et al. Factors affecting long-term postoperative narcotic use in discectomy patients. World Neurosurg. 2018;112:e640-e644.

Disclosures and COI: The authors declare that there are no relevant conflicts of interest to disclose.

Corresponding Author: Chad M. Patton, MD, MS, Center for Spine Surgery, Anne Arundel Medical Center, 2000 Medical Parkway, Suite 101, Annapolis, MD 21401. Phone: (443) 481-1000; Email: cpatton@aahs.org.

Published 30 April 2020

This manuscript is generously published free of charge by ISASS, the International Society for the Advancement of Spine Surgery. Copyright (c) 2020 ISASS. To see more or order reprints or permissions, see http://ijssurgery.com. 